\title{
A Difficulty Chart for Estimation of Treatment Duration of Palatally Impacted Canines
}

\author{
Riyadh A. Ruwiaee ${ }^{1}$, Dhuha A. Al-Ali², Mushriq F. Abid ${ }^{3}$ \\ ${ }^{1}$ BDS, MSc/ Post Graduate/ ${ }^{2}$ BDS, MSc / post graduate, ${ }^{3}$ BDS, MSc, PhD Assist Prof. / Department of \\ Orthodontics/College of Dentistry/University of Baghdad
}

\begin{abstract}
Treatment of maxillary impacted canine is a challenging process to dental specialists and orthodontists. The aim of the present study was to assess the duration of orthodontic treatment of palatally impacted canines, using a difficulty chart as an estimate of the prognosis, and relating it to the treatment time and to other factors that might affect the treatment duration. A cross-sectional study was undertaken using a selfadministered questionnaire to be completed by orthodontists ( 82 participants). An index was used as a tool to assess the prognosis and treatment duration. The study found that $48.7 \%$ of active orthodontic treatment time for palatally impacted canines was completed in 1.5 to 2 years. The number of active treatment visits per patient was between 16 and 22 (62\% of treatment visits). In terms of the treatment performed, $81.7 \%$ of cases had space created prior to surgical exposure, and 59.8\% had open surgical exposure. Failed to attend visits by the patient accounted for $48.4 \%$ and $62.2 \%$ did not require repeated surgical exposure. Interestingly, $85 \%$ of the cases had 'good' or 'average' prognosis ( $49 \%$ and $36 \%$, respectively). A prognostic chart can provide an estimate for the treatment time to bring a palatally impacted canine into the arch. This information might be valuable for both orthodontists and their patients, to estimate the time of the planned treatment and provide a valid consent form.
\end{abstract}

Keywords: Palatally impacted canine; Treatment time; Difficulty chart, Surgical exposure.

\section{Intoduction}

The maxillary canine plays a major role in the aesthetic and functional aspects of the occlusion and its absence should be avoided wherever possible. ${ }^{[1]}$ This tooth is considered as the most frequently affected tooth with eruption problems, following the third molar, with a reported prevalence of $0.92 \%$ to $6.04 \% .{ }^{[2,3]}$ Data from previous studies suggests that, $85 \%$ of canine impactions occur palatally and $15 \%$ buccally, moreover $8 \%$ to $10 \%$ of canine impactions occur bilaterally. ${ }^{[4]}$

Several studies have linked canine impaction with multiple etiological factors such as anomalies and absence of lateral incisors, ectopic tooth germ position, the presence of mechanical obstruction, and genetic

\section{Corresponding Author:}

Name: Mushriq F. Abid

E-mail: mushriq.abid@codental.uobaghdad.edu.iq factors. ${ }^{[5]}$ The treatment of palatally impacted canines is a common challenge faced by dental professionals in daily practice. Different types of treatment modalities have been suggested, including early interceptive or late management, that combines surgical-orthodontic methods. ${ }^{[6,7]}$ Canine extraction and auto-transplantation have been suggested as an alternative way of management. ${ }^{[8,9]}$ Special biomechanics and anchorage are always required during canine traction, such as large cantilever wires with adequate support for the adjacent teeth. ${ }^{[10,11]}$

The active orthodontic treatment time is defined in relation to either the bonding of a fixed orthodontic appliance or the final realignment of the impacted canine within the dental arch. Published data shows the overall treatment time ranging from 19.6 to 28.8 months, with large individual variations. ${ }^{[12]}$ The number of orthodontic dental visits required to treat ectopic maxillary canines in past studies has varied from 17.7 to 
39.8 appointments. ${ }^{[12,13]}$

Any treatment of palatally impacted canine is considered complex. ${ }^{[14]}$ This complexity depends on several factors including the location (palatal, buccal), the angle of impaction, and how close the canine is to the midline. ${ }^{[15]}$ Other factors that might complicate and increase the treatment time include unfavourable impaction, the method of exposure and traction, whether space has been created pre-exposure, the age of the patient, and appointment numbers relating to active orthodontic treatment. ${ }^{[16]}$ Several classifications have been advocated to quantify the severity of canine impaction and to estimate the prognosis, considering how complex the treatment of each case could be. ${ }^{[17,18]}$ According to the Ericson and Kurl classification, an impaction close to the midline is considered complex and requires special biomechanics. ${ }^{[19]}$ Similarly, a horizontally impacted canine (angle of impaction) is more challenging and may compromise the prognosis. ${ }^{20]}$ Information about orthodontic treatment time and prognosis needs to be given to patients when discussing treatment options in order to obtain valid informed consent.

To our knowledge, no previous study in Iraq has addressed the prognosis and the actual treatment time needed to bring the canine into the dental arch. This study sets out to assess the treatment difficulty and duration of palatally impacted canines, with the help of an index (chart) especially used to estimate the prognosis of treatment.

\section{Methods}

This is a cross-sectional descriptive study designed with the aid of a self-administered questionnaire. The study was conducted in the College of Dentistry (Baghdad University) from April 2019 till October 2019. The study population included orthodontist specialists working at academic institutions, private clinics, and hospitals: all were invited to participate in the study. Overall, a total of 120 surveys were distributed, of which 38 were excluded as they were inappropriately filled in or incomplete; in the end, only 82 questionnaires were eligible for data analysis. The study was approved by a local committee in the Orthodontic Department at the College of Dentistry (Baghdad University).
This survey, in the form of a questionnaire, focused only on patients with palatally impacted canines (unilateral or bilateral) who had received treatment from the orthodontists. For example, one case was selected in which the canine was tracked to its ideal position in the arch using a fixed appliance; this case was chosen by the orthodontist to be one of the best cases that was treated, and the treatment was completed on time. It involved the selection of the prognostic factors and the canine position relating to the treated case, using the parameters from previous studies. ${ }^{[21,22]}$ A chart recommended by the Royal College of Surgeons (RCS Eng) guidelines was given to the orthodontists to estimate treatment difficulty and prognosis ${ }^{[17]}$, as shown in Figure 1. The prognosis considered 'good' was when all four categories were good, 'average' prognosis was considered when only two categories were 'good'. 'Poor' prognosis in all of the categories was considered to be 'poor'.

The questionnaire involved several questions in the form of closed questions, such as the active orthodontic treatment time was specified as the time from the bonding to the debonding of the fixed appliance, and the time needed to realign the canine to correct occlusion (from exposure time to the dental arch) to bring the canine in occlusion. Other details were recorded, including: the total number of active visits, the orthodontic anchorage devices used, the time taken for canine realignment from exposure to correct occlusion, the radiographic assessment, age and gender of the patient, whether the impaction was unilateral or bilateral, the method of exposure and mechanical traction, pre or post exposure space creation, number of failures, and emergency visits and failed to attend visits during the course of the orthodontic treatment (Table 1). The survey was piloted by ten orthodontists to assess the suitability of the questions which were reviewed and adjusted to ensure scientific accuracy. The data was analysed using Microsoft Excel starter 2010 (Microsoft Corporation).

\section{Results}

Data from 82 orthodontists for 82 patients with palatally impacted canines were collected and analysed with a response rate of $68.3 \%$. About two-thirds of the patients were females $(65.9 \%)$, and the age range of the patients was 12 to 20 years (mean $=14$ years old). Unilateral canine impaction accounted for $69.5 \%$ of the 
cases.

The survey revealed that $49 \%$ of active orthodontic treatment for palatally impacted canines was completed in 1.5 to 2 years, with a mean of 21 months (Figure 2A). Sixty-eight percent of the patient's canine realignment (from exposure to the correct occlusion) was achieved in 15 months (range of 12 to 18 months). Sixty-two percent of active treatment visits made by the patients were between 16 to 22, with a mean of 19 visits (Figure 2B).

In $81.7 \%$ of the cases, space for the impacted canine was created prior to exposure, and in 59.8\% open surgical exposure was performed (Figure $3 \mathrm{~A}$ ). The number of visits failed to attend by the patient during the treatment was $48.8 \%$ (less than four visits) (Figure 3B), and $62.2 \%$ of the patients did not require additional or repeated surgical exposure (Figure 3C).

Regarding the estimation of treatment difficulty, using the chart provided, four categories were included: incisor overlap, vertical height, and the angulation and position of the apex. The results revealed that $51.3 \%$ of cases had a 'good' prognosis, and 34.1\% had an 'average' prognosis, while $14.6 \%$ of cases had a 'poor' prognosis (Figure 4).

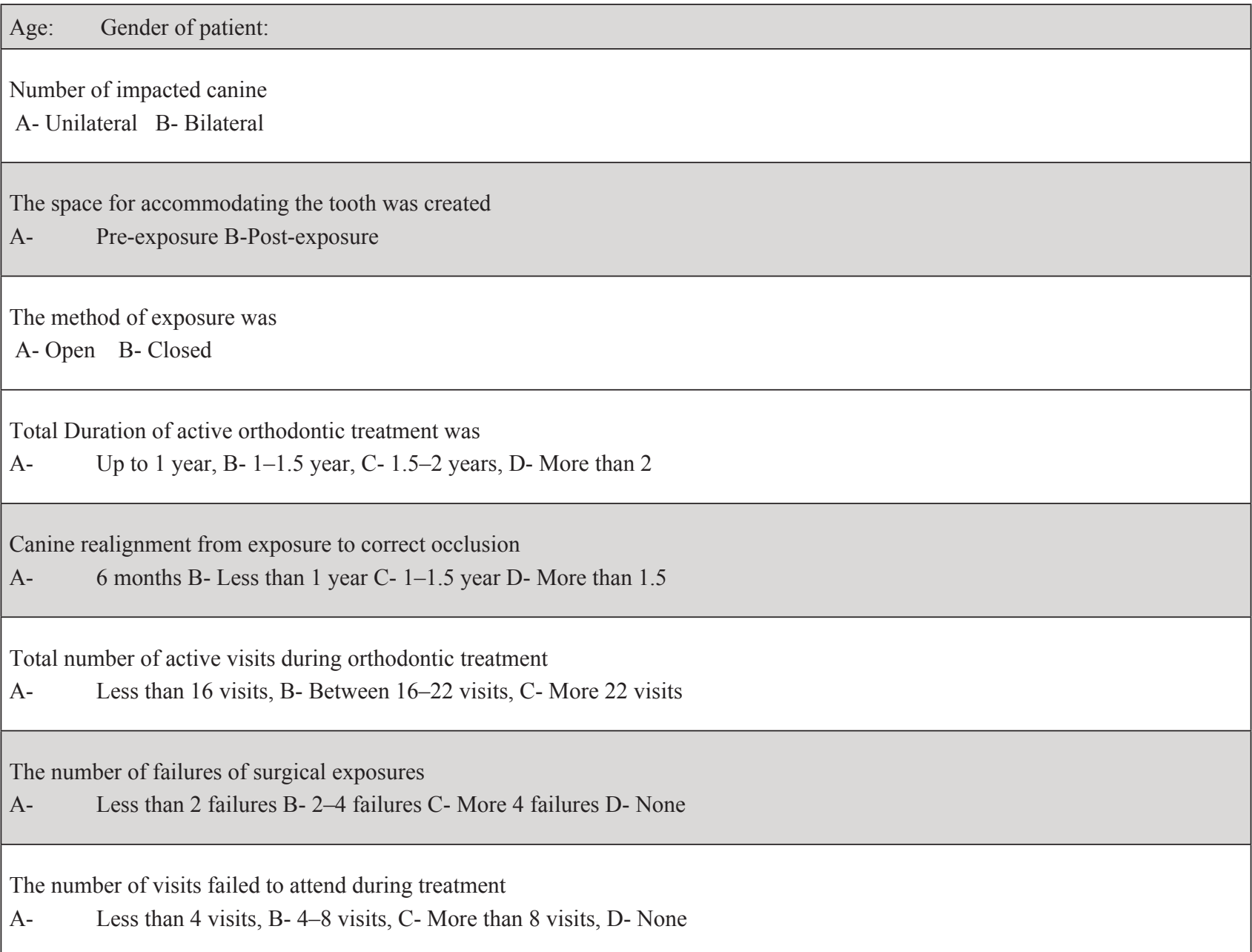


Table 1: Questionnaire for data collection.

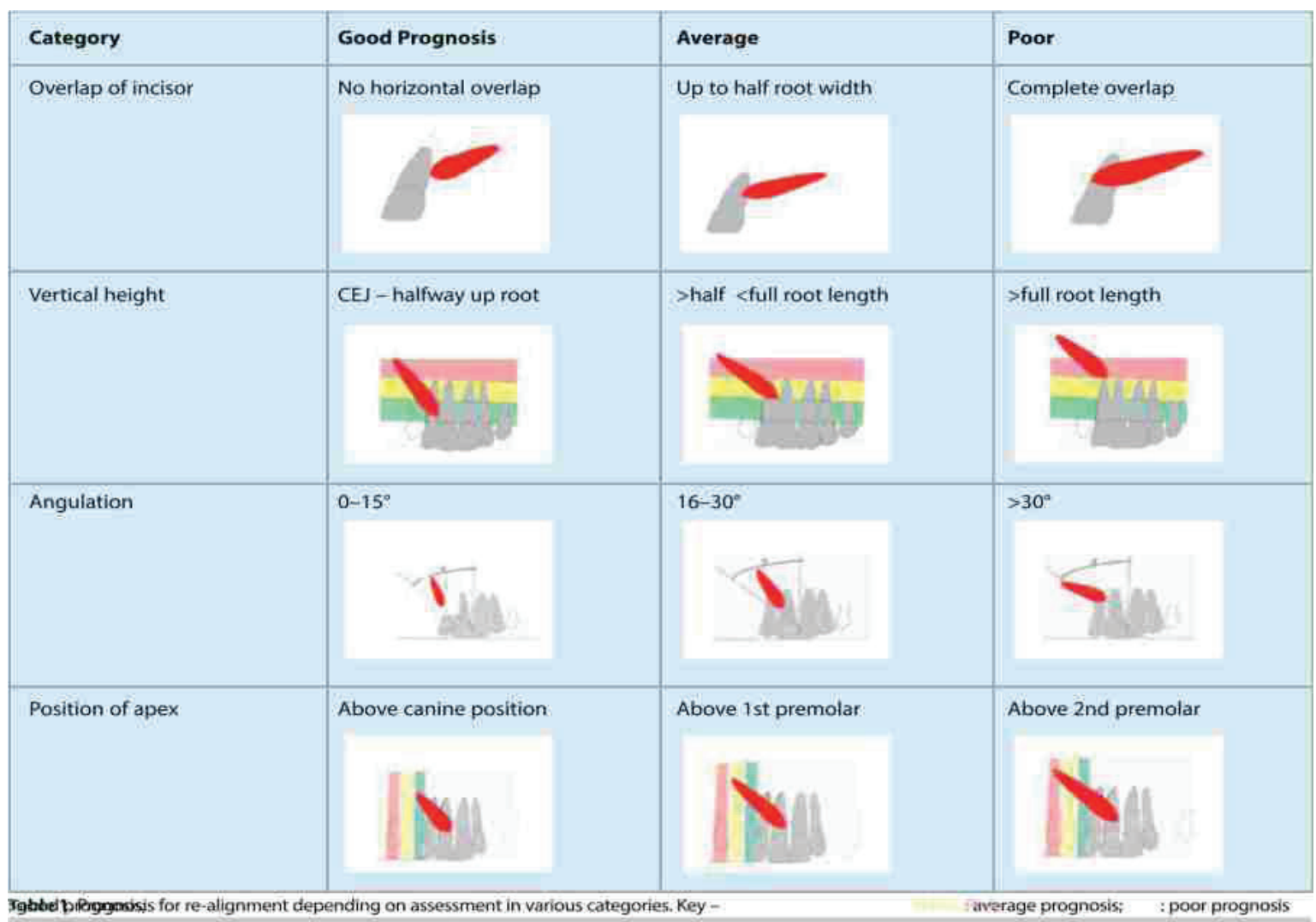

Figure 1: Prognosis for realignment depending on assessments across various categories. Key - Green = good prognosis; Yellow $=$ average prognosis; Pink $=$ poor prognosis. adapted from. ${ }^{[17]}$
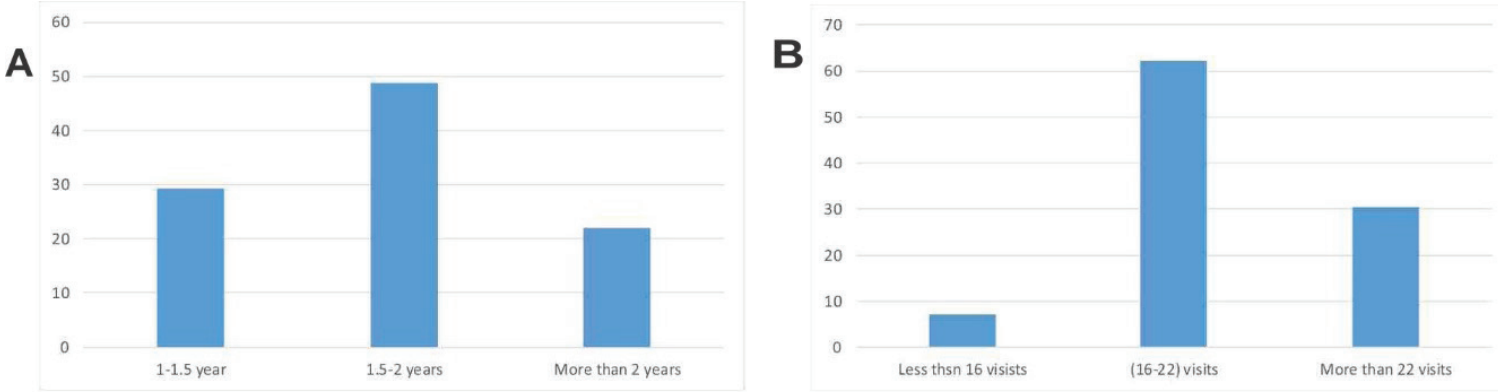

Figure 2: A) The duration of active orthodontic treatment, B) the number of visits attended by the patient. 


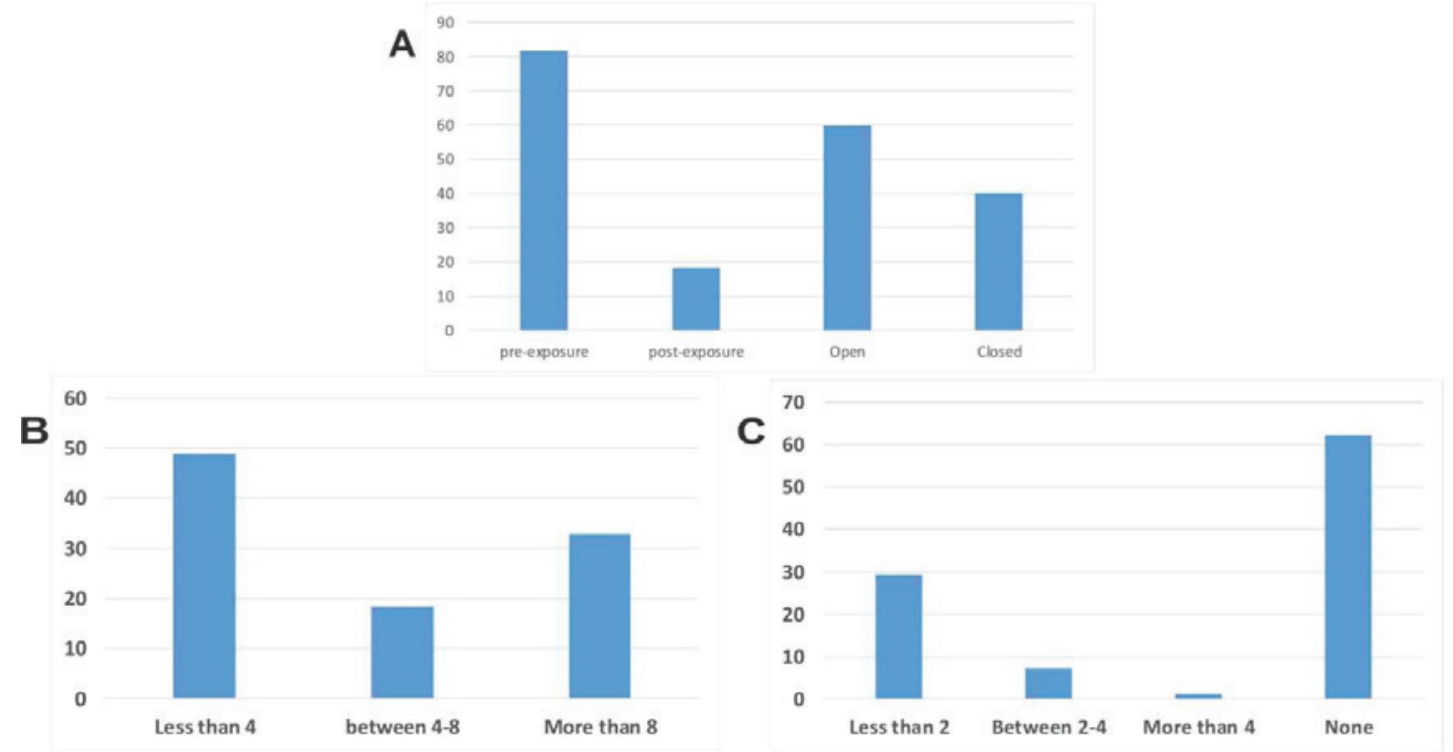

Figure 3: A) Space creation and type of surgical exposure B) Failed surgical exposure C) Visits failed to attend by the patient.

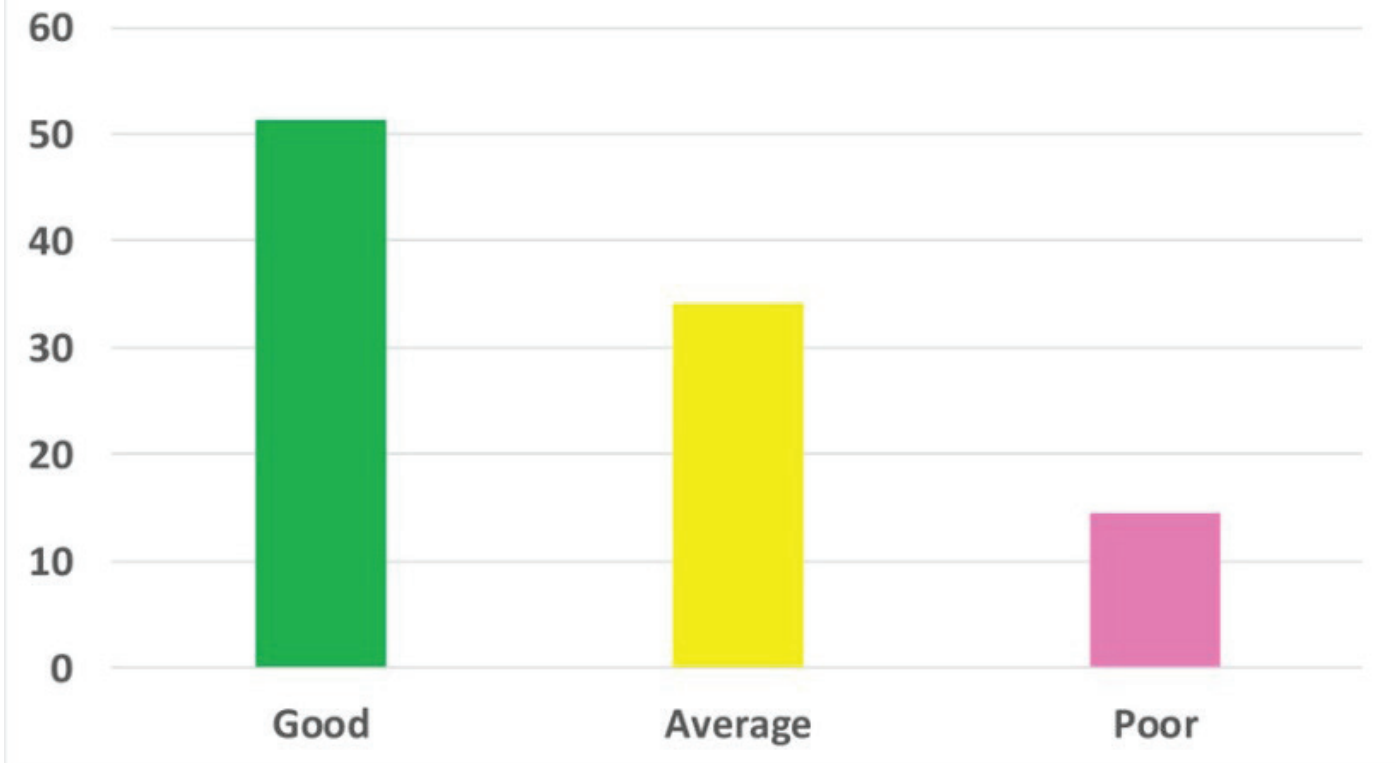

Figure 4: Prognosis assessment using the chart (\%).

\section{Discussion}

The orthodontic management of palatally impacted canine requires an accurate assessment of the canine location in different dimensions. Accurate localisation helps to surgically expose and retrieve the tooth efficiently and individualise the clinical approach and biomechanics. Both patients and orthodontists need to know the expected treatment duration, which, to a great extent, is affected by the location of the canine. It has previously been observed that the duration of treatment is affected by several factors, including the number of missed appointments, the number of debonded brackets and bands, poor oral hygiene, the number of extracted bicuspid, mandibular plane angle, and age at the start of treatment. ${ }^{[23-25]}$

Various treatment difficulty indices have been proposed to estimate the severity of impaction. However, this study used a chart that has been recommended by the RCS-Eng guidelines for the management of palatally impacted canines. Four aspects of canine position were assessed, including incisor overlap, vertical height, the 
angulation, and the position of the apex. Data from published studies suggest that the treatment duration of palatally impacted canine lasts for an average of 18 to 30 months. ${ }^{[13,26,27]}$ Turning now to the results recorded in the present study, the average active orthodontic treatment time was 21 months, which falls within the range of the studies mentioned previously. The time needed to bring the canine from its location at the time of surgical exposure to the line of the dental arch was about 15 months in the majority of cases (68\%).

The average total number of active orthodontic visits was 19 visits which falls within the same range found by other studies (17.7 to 39.8) visits. ${ }^{[22,28,29]}$ In response to the prognosis, up to half of the cases $(48.7 \%)$ had a 'good' prognosis while $36 \%$ had 'average' prognosis. This makes a total of $85 \%$ of the reported cases who had 'good' and 'average' prognosis. In most of these cases, the treatment time was around 21 months (between 1.5 to 2 years) which might indicate that the chart can be used as a useful tool to estimate the treatment duration. Conversely, other cases within the same categories showed a longer treatment time, which could be related to other factors, including the orthodontist's experience, mechanics, and age of the patients. Another possible explanation for finishing these cases within the expected duration could be attributed to results found in the present study. For example, for $81.7 \%$ of cases, the space was created prior to surgical exposure, $62.2 \%$ did not have to repeat the surgical exposure, and $48.4 \%$ had less than four visits which they failed to attend. Interestingly, these factors were previously emphasised by previous published data as contributing factors adding extra time to bring the impacted tooth into the arch. ${ }^{[15,28-30]}$

The limitations of the study were that only the besttreated case by each orthodontist was included and it did not involve different types of cases. Additionally, no inferential statistics were made in the present study, which would be more feasible if more cases and different groups were involved.

\section{Conclusions}

On the basis of the results of this study, a rough prediction for the treatment of palatally impacted canine can be made using a difficulty chart. This chart might be helpful in providing information to the patient to estimate the treatment duration. Further studies are required to include other variables to improve the accuracy of the time needed to complete the treatment.

Ethical Clearance: The Research Ethical Committee at scientific research by ethical approval of both $\mathrm{MOH}$ and MOHSER in Iraq

\section{Conflict of Interest: Non}

Funding: Self-funding

\section{References}

1. Armi P, Cozza P, Baccetti T. Effect of RME and headgear treatment on the eruption of palatally displaced canines: A randomized clinical study. Angle Orthod. 2011;81(3):370-374.

2. Herrera-Atoche JR, Agüayo-De-Pau MDR, Escoffié-Ramírez M, Aguilar-Ayala FJ, CarrilloÁvila BA, Rejón-Peraza ME. Impacted Maxillary Canine Prevalence and Its Association with Other Dental Anomalies in a Mexican Population. Int J Dent. 2017;2017.

3. D’Oleo-Aracena MF, Arriola-Guillén LE, Rodríguez-Cárdenas YA, Ruíz-Mora GA. Skeletal and dentoalveolar bilateral dimensions in unilateral palatally impacted canine using cone beam computed tomography. Prog Orthod. 2017;18(1).

4. Ericson S, Kurol J. Longitudinal study and analysis of clinical supervision of maxillary canine eruption. Community Dent Oral Epidemiol. 1986;14(3):172176.

5. Peck S, Peck L, Kataja M. The palatally displaced canine as a dental anomaly of genetic origin. Angle Orthod. 1994;64(4):249-256.

6. Caprioglio A, Bergamini C, Franchi L, et al. Prediction of Class II improvement after rapid maxillary expansion in early mixed dentition. Prog Orthod. 2017;18(1).

7. Pisani L, Bonaccorso L, Fastuca R, Spena R, Lombardo L, Caprioglio A. Systematic review for orthodontic and orthopedic treatments for anterior open bite in the mixed dentition. Prog Orthod. 2016;17(1).

8. Berglund L, Kurol J, Kvint S. Orthodontic pretreatment prior to autotransplantation of palatally impacted maxillary canines: Case reports on a new approach. Eur J Orthod. 1996;18(5):449-456.

9. D’Amico RM, Bjerklin K, Kurol J, Falahat B. Longterm results of orthodontic treatment of impacted 
maxillary canines. Angle Orthod. 2003;73(3):231238.

10. Oppenhuizen. An extrusion spring for palatally impacted cus- pids. J Clin Orthod. 2003;37:434436.

11. Thebault B, Dutertre E. Disimpaction of maxillary canines using temporary bone anchorage and cantilever springs. Int Orthod. 2015;13(1):61-80.

12. Becker A, Chaushu S. Success rate and duration of orthodontic treatment for adult patients with palatally impacted maxillary canines. Am J Orthod Dentofac Orthop. 2003;124(5):509-514.

13. Pearson MH, Robinson SN, Reed R, Birnie DJ, Zaki GA. Management of palatally impacted canines: The findings of a collaborative study. Eur J Orthod. 1997;19(5):511-515.

14. Nakandakari C, Gonçalves JR, Cassano DS, Raveli TB, Bianchi J, Raveli DB. Orthodontic Traction of Impacted Canine Using Cantilever. Case Rep Dent. 2016;2016.

15. Cuminetti F, Boutin F, Frapier L. Predictive factors for resorption of teeth adjacent to impacted maxillary canines. Int Orthod. 2017;15(1):54-68.

16. Stivaros N, Mandall NA. Radiographic factors affecting the management of impacted upper permanent canines. J Orthod. 2000;27(2):169-173.

17. Counihan K, Al-Awadhi EA, Butler J. Guidelines for the assessment of the impacted maxillary canine. Dent Update. 2013;40(9):770-777.

18. Kau CH, Lee JJ, Souccar NM. The validation of a novel index assessing canine impactions. Eur J Dent. 2013;7(4):399-404.

19. Ericson S, Kurol J. Radiographic examination of ectopically erupting maxillary canines. Am J Orthod Dentofac Orthop. 1987;91(6):483-492.

20. Arriola-Guillén LE, Ruíz-Mora GA, RodríguezCárdenas YA, Aliaga-Del Castillo A, BoessioVizzotto M, Dias-Da Silveira HL. Influence of impacted maxillary canine orthodontic traction complexity on root resorption of incisors: A retrospective longitudinal study. Am J Orthod Dentofac Orthop. 2019;155(1):28-39.
21. McSherry P. The assessment of and treatment options for the buried maxillary canine. Dent Update. 1996;23(1):7-10.

22. Husain MJ, Burden D, McSherry MP. The management of the palatally ectopic maxillary canine: 2004. R Coll Surg England, Fac Dent Surg Clin Guidel. 2004. http://www. taysideorthodonticmen.scot.nhs.uk/documents/ guidelines/management_of_palatally_ectopic_ maxillary_canine.pdf.

23. Alger D. Appointment frequency versus treatment time. Am J Orthod Dentofac Orthop. 1988;94:436439.

24. Beckwith R, Ackerman R, Cobb C, Tira D. An evaluation of factors affecting duration of orthodontic treatment. Am J Orthod Dentofac Orthop. 1999;115:439-447.

25. Fink D, Smith R. The duration of orthodontic traetment. Am J Orthod Dentofac Orthop. 1992;102:45-51.

26. Becker A, D K, Ziberman Y. Periodontal status following the alignment of palatally impacted canine teeth. Am J Orthod. 1983;84:332-336.

27. Iramaneerat $S$, Cuningham S, Horrocks E. The effect of two alternative methods of canine exposure upon subsequent duration of orthodontic treatment. Int J Pediatr Dent. 1998;8:123-129.

28. Zuccati G, Ghobadlu J, Nieri M, Clauser C. Factors associated with the duration of forced eruption of impacted maxillary canines: A retrospective study. Am J Orthod Dentofac Orthop. 2006;130(3):349356.

29. Larsen HJ, Sørensen HB, Artmann L, Christensen IJ, Kjær I. Sagittal, vertical and transversal dimensions of the maxillary complex in patients with ectopic maxillary canines. Orthod Craniofacial Res. 2010;13(1):34-39.

30. Stewart JA, Heo G, Glover KE, Williamson PC, Lam EWN, Major PW. Factors that relate to treatment duration for patients with palatally impacted maxillary canines. Am J Orthod Dentofac Orthop. 2001;119(3):216-225. 